\title{
Effects of honey supplementation on inflammatory markers among chronic smokers: a randomized controlled trial
}

\author{
Wan Syaheedah Wan Ghazali, Aminah Che Romli and Mahaneem Mohamed*
}

\begin{abstract}
Background: Honey has been demonstrated to possess anti-inflammatory property. This is a randomized, controlled, open-label trial to determine the effects of 12-week honey oral supplementation on plasma inflammatory markers such as high sensitive C-reactive protein, interleukin-6 and tumor necrosis factor-a among chronic smokers.

Methods/design: A total of 32 non-smokers and 64 chronic smokers from Quit Smoking Clinic and Health Campus, Universiti Sains Malaysia participated in the study. Smokers were then randomized into 2 groups: smokers with honey group that received Malaysian Tualang honey ( $20 \mathrm{~g} /$ day daily for 12 weeks) and smokers without honey group. Blood was obtained from non-smokers and smokers at pre-intervention, and from smokers at postintervention for measurement of the inflammatory markers.
\end{abstract}

Results: At pre-intervention, smokers had significantly higher high sensitive C-reactive protein than non-smokers. In smokers with honey group, tumor necrosis factor-a was significantly increased while high sensitive C-reactive protein was significantly reduced at post-intervention than at pre-intervention.

Conclusion: This study suggests that honey supplementation has opposite effects on tumor necrosis factor-a and high sensitive C-reactive protein indicating the inconclusive effect of honey on inflammation among chronic smokers which needs further study on other inflammatory markers.

Trial registration: The Trial has been registered in the Australian New Zealand Clinical Trials Registry: ACTRN12615001236583. Registered 11 November 2015 (Retrospectively Registered).

Keywords: Chronic smokers, Honey, Inflammatory markers, C-reactive protein, Tumor necrosis factor-a

\section{Background}

Cigarette smoking has been reported as one of the major causes of cardiovascular disease [1]. Elevated inflammation in smokers has been proposed as one of the mechanisms involved in initiating and accelerating the atherothrombotic process leading to cardiovascular disease development. Several studies reported that cigarette smoke exposure is associated with increased inflammation. It has been found that the level of serum inflammatory marker such as tumor necrosis factor- $\alpha$ (TNF- $\alpha)$ is significantly higher in smokers compared to non-smokers [2]. Quit smoking is the

* Correspondence: mahaneem@usm.my

Department of Physiology, School of Medical Sciences, Universiti Sains Malaysia, 16150 Kubang Kerian, Kelantan, Malaysia best method to reduce the risk of cardiovascular disease. However, there is still a need to reduce this risk among chronic smokers who fail to quit smoking which in turn leads to an increased interest to investigate the possible beneficial effect of natural products among smokers. Previous study has reported that supplementation of camu-camu juice (with high vitamin $\mathrm{C}$ content), which has anti-inflammatory activity, among male smokers for 7 days significantly reduces the levels of serum high sensitivity C-reactive protein (hsCRP), interleukin-6 (IL-6) and interleukin-8 (IL-8) [3].

Honey is a natural product of bees and contains carbohydrates such as glucose and fructose as well as proteins, minerals, organic acids and aromatic compounds [4]. Apart from that, it also contains enzymes such as 
glucose oxidase and catalase [5], vitamins $\mathrm{A}$ and $\mathrm{E}$ [6], flavonoids [7, 8], phenolic acids [9], as well as has antioxidant properties [10]. Honey has been demonstrated to possess a potent anti-inflammatory property. It is suggested that protection of murine keratinocytes from ultraviolet radiation-induced inflammation by honey added to cell cultures occurs through the suppression of inflammatory cytokines, ultraviolet B-induced cyclooxygenase-2 expression, inducible nitric oxide synthase protein expression as well as through the production of prostaglandin $\mathrm{E}_{2}$ [11]. In animal study, oral treatment with $5 \mathrm{~g} / \mathrm{kg}$ and $10 \mathrm{~g} / \mathrm{kg}$ of honey has been shown to reduce the inflammation in an experimentally-induced inflammatory bowel disease in rats [12]. Meanwhile, in human study, honey supplementation at a dose of $70 \mathrm{~g}$ daily for 8 weeks significantly reduces seminal plasma inflammatory markers such as TNF- $\alpha$, IL-6 and IL-8 among male road cyclists [13].

However, to date, no study has been reported to determine whether honey supplementation is able to improve inflammation among smokers. Therefore, the aim of this study was to determine the possible role of honey in improving inflammation among chronic smokers.

\section{Methods}

\section{Subjects and eligibility}

This study involved subjects aged between 20 and 50 years old. Subjects from non-smoker group should have no history of smoking and exposure to environmental tobacco smoke. Meanwhile, subjects from smoker group should have smoked at least 10 cigarettes per day for more than 5 years. Exclusion criteria were the presence of severe infection, regular consumption of dietary supplements and/or multivitamins 3 months prior to the study, obese $\left(\mathrm{BMI}>30 \mathrm{~kg} / \mathrm{m}^{2}\right)$, taking alcohol or had history of cardiovascular disease. Ethical approval was obtained from Human Research Ethics Committee of Universiti Sains Malaysia (USM) (approval code: JEPeM [243.3.(6)]. This study took place in Physiology Laboratory, School of Medical Sciences, USM from September 2012 to August 2014.

\section{Sample size}

Sample size for each group was calculated using Power and Sample Size Calculation Software version 3.0.10. The assumption of Type I error probability was 5\% $(0.05)$ while the power of the study was $80 \%(0.8)$. The difference in population means between two groups $(\delta)$ was $30.2 \mathrm{pg} / \mathrm{mL}$ and within group standard deviation $(\sigma)$ was $40.1 \mathrm{pg} / \mathrm{mL}$ based on IL-6 level from the previous study [13]. The ratio of control to experimental group (m) was standardized at 1 . Considering a drop-out rate of $20 \%, 36$ subjects were recruited for each smoker group of the study. However, the final number of subjects for each group was 32 after excluding the dropped out subjects.

\section{Study design and intervention}

In this an open-label randomized controlled trial, a total of 108 subjects from USM staff in the Health Campus and Quit Smoking Clinic, USM Hospital were screened for inclusion in the study. Thirty eight from them were screened for non-smoker group but 6 subjects were excluded because of not meeting the inclusion criteria. Meanwhile, 80 subjects were screened for smoker group and 8 subjects were excluded because of not meeting the inclusion criteria. The objectives of the study were carefully explained to the subjects and informed consent was taken. Blood was obtained from all subjects (nonsmokers and smokers) during the initial visit (week 0) for the pre-intervention status assessment. Smokers from smoker group were then assigned into 2 parallel groups namely smokers with honey $(n=36)$ and smokers without honey $(n=36)$. Simple randomization was done by the first author using a randomized table created by computer software (Random allocation software version 1.0). The allocation was not concealed and allocation ratio was 1:1. Supplementation of honey ( $20 \mathrm{~g} /$ day orally for 12 weeks) was given to the smokers with honey group at weeks 0,4 and 8 . Honey used in this study was a pure local honey named Tualang honey supplied by Federal Agricultural \& Agro Based Industry, Malaysia. The subjects were instructed to return the empty sachet to ensure compliance and any possible side effects of supplementation were monitored. During the study, 4 subjects refused to continue the intervention and the final number of subjects was 32 in smokers with honey group $(n=32)$. Similarly, 4 subjects refused to continue the intervention and the final number of subjects was 32 in smokers without honey group $(n=32)$. Post-intervention status was reassessed after 12 weeks. As for the subject from non-smoker group, blood was obtained only at pre-intervention for baseline comparison on the status of inflammatory markers between non-smoker and smoker groups. All subjects were asked to maintain their current activity levels, which include diet and exercise, during the study. The flowchart of this study is shown in Fig. 1.

\section{Blood collection and sample preparation}

A total of $3 \mathrm{~mL}$ of venous blood was obtained from each subject at pre-intervention and from each subject of both smoker groups at post-intervention. The blood was collected into a tube containing ethylenediamine tetraacetic acid. The whole blood in the tube was centrifuged at $1000 \mathrm{x} \mathrm{g}$ for $10 \mathrm{~min}$ at $4{ }^{\circ} \mathrm{C}$. A hundred $\mu \mathrm{L}$ of plasma was alliquoted into each microcentrifuge tube and kept 


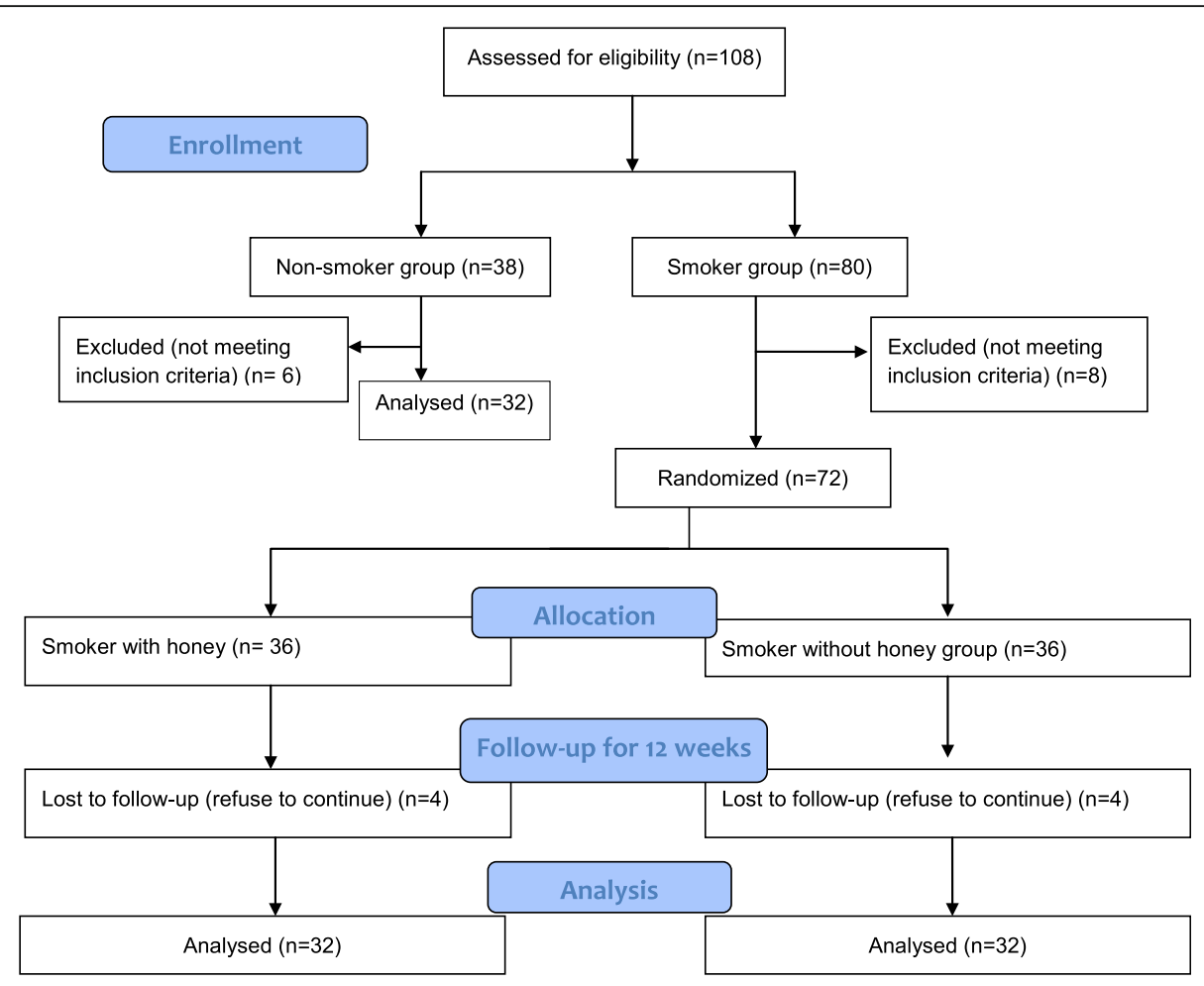

Fig. 1 CONSORT Flow diagram of subject recruitment

frozen at $-80{ }^{\circ} \mathrm{C}$ for biochemical analysis of plasma inflammatory markers which include TNF- $\alpha$, IL- 6 and hsCRP.

\section{Determination of inflammatory markers}

Plasma TNF- $\alpha$, IL- 6 and hsCRP were determined using Human TNF-alpha ELISA Kit (Raybiotech, USA), Human Interleukin 6 ELISA Kit (AssayPro, USA) and Human hs-CRP ELISA Kit (Cusabio, USA), respectively.

\section{Statistical analysis}

Results were analysed using Statistical Package for the Social Sciences version 20. Independent $t$-test was used to analyse the difference of pre-intervention inflammatory markers between non-smoker and smoker groups. The differences between pre and post-intervention inflammatory markers in each group of smokers were assessed by paired $t$-test. A value of $p<0.05$ was considered statistically significant. Data are presented as mean and standard error of mean (SEM).

\section{Results}

Table 1 shows the baseline characteristics of the subjects. There were no significant differences for mean age and mean arterial pressure (MAP) between nonsmoker and smoker groups.
Pre-intervention level and changes (between pre and post-intervention) of inflammatory markers among subjects

The levels of inflammatory markers at pre-intervention are presented in Table 2. The levels of mean plasma TNF- $\alpha$ and mean plasma IL- 6 at pre-intervention did not show any significant differences between smoker and non-smoker groups. In contrast, the level of mean plasma hsCRP at pre-intervention was significantly higher in smoker group compared to non-smoker group.

The levels of inflammatory markers at post-intervention are presented in Table 3. At post-intervention, the mean plasma TNF- $\alpha$ was significantly increased while the mean plasma hsCRP was significantly reduced after 12 weeks in smokers with honey group. No significant differences were observed for the levels of mean plasma TNF- $\alpha$ and mean plasma hsCRP in smokers without honey group. Furthermore, the level of mean plasma IL-6 revealed no

Table 1 Characteristics of the subjects between non-smokers and smokers groups

\begin{tabular}{lll}
\hline & Non-smokers $(n=32)$ & Smokers $(n=64)$ \\
\hline Age (years) & $35.16(1.50)$ & $38.38(0.97)$ \\
MAP $(\mathrm{mmHg})$ & $89.77(0.99)$ & $92.57(1.06)$ \\
\hline
\end{tabular}

Data are presented as mean (SEM). MAP: Mean arterial pressure. No significant differences for mean age and mean arterial pressure (MAP) between nonsmokers and smokers groups (Independent $t$-test) 
Table 2 Pre-intervention inflammatory markers between nonsmokers and smokers groups

\begin{tabular}{lll}
\hline & Non-smokers $(n=32)$ & Smokers $(n=64)$ \\
\hline TNF- $a(\mathrm{pg} / \mathrm{mL})$ & $2755.42(172.00)$ & $2821.25(99.71)$ \\
IL-6 $(\mathrm{ng} / \mathrm{mL})$ & $2.37(0.30)$ & $1.77(0.16)$ \\
hsCRP $(\mathrm{ng} / \mathrm{mL})$ & $1.58(0.25)$ & $2.45(0.24)^{*}$
\end{tabular}

Data are presented as mean (SEM). TNF-a: tumor necrosis factor-alpha; IL interleukin; $h s C R P$ high sensitivity C-reactive protein. ${ }^{*} p<0.05$ compared to non-smokers (Independent $t$-test)

significant difference in both smokers with honey and smokers without honey groups.

\section{Discussion}

Inflammation is fundamental to the body's defence against infection. Furthermore, it is also a critical component of normal tissue repair. Cigarette smoking has been shown to alter the host response and therefore, modifies the progression and outcome of inflammation. The exact mechanisms of cigarette smoking leading to inflammation are not clearly understood. However, it has been postulated that smoking affects a number of inflammatory process through its effect on immuneinflammatory cells, causing an immunosuppressant state and cytokine secretion [14]. Therefore, in this study, the levels of inflammatory markers such as TNF- $\alpha$, IL-6 and hsCRP were measured in plasma of smokers with and without honey supplementation. Inflammatory markers were also measured at pre-intervention among nonsmokers for baseline comparison.

The level of TNF- $\alpha$ at pre-intervention in smoker group was not statistically significant compared to nonsmoker group which might suggest that macrophages are not activated to produce TNF- $\alpha$ among smokers in the present study. However, the level of plasma TNF- $\alpha$ revealed a significant increase after 12 weeks of honey supplementation. This result is in accordance with previous in vitro study which shows that $5.8 \mathrm{kDA}$ protein component of honey is able to stimulate the production of TNF- $\alpha$ by macrophages via toll-like receptor (TLR)-4. TLR-4 is able to detect lipopolysaccharides from gramnegative bacteria, thus, plays a role in pathogen recognition and activation of innate immunity. In human monocytes, blocking of the TLR-4 but not TLR-2 receptor significantly inhibits honey-stimulated TNF- $\alpha$ production, thus, suggesting TLR-4 receptor involvement [15]. A family of TLRs serves as primary sensors that recognize a variety of microbial components as well as induce innate immune responses. All pathways of TLR signaling terminate in NF-kB (nuclear factor kappa-light-chain-enhancer of activated $B$ cells activation), a protein complex that controls the inflammatory cytokine genes expression [16]. Therefore, activation of NF-kB may lead to an increase in expression of inflammatory cytokines which include TNF- $\alpha$, IL-6, IL-8, IL-12 and IL-18 [14]. In the present study, it is therefore plausible to suggest that honey may contribute in activation of NF-kB of macrophages via TLRs leading to increased expression and production of TNF- $\alpha$. However, the finding on the increased TNF- $\alpha$ level seems to suggest that honey may exert an inflammatory effect, but it needs further study by also measuring other inflammatory markers such as IL-1, IL-4 and IL-10.

The level of plasma IL- 6 in the present study was slightly lower in smoker group compared to non-smoker group at pre-intervention but not statistically significant. On the contrary, in other study, it is found that there is a significantly higher level of serum IL-6 among smokers compared to non-smokers. The increased level of serum IL-6 indicates a higher level of cytokine-mediated inflammation among elderly active smokers (mean age of 77 years) [17]. These conflicting results may be due to the younger age group (mean age of 38 years) of the subjects involved in the present study or due to the relatively small sample size of the present study. Following 12 weeks of honey supplementation, the level of plasma IL-6 was found to be slightly higher but not statistically significant. However, previous study has shown that honey stimulates IL-6 secretion from human monocytes, leading to an activation of immune response in the cell [18]. Furthermore, honey supplementation at dose of $0.27 \mathrm{ml} / \mathrm{kg} /$ orally for 7 days significantly increases the level of mRNA IL-6 expression in Salmonella typhi induced of mice [19]. Apart from that, in mice infected with invasive aspergillosis, 10 days honey supplementation at a dose of $1.5 \mathrm{~g} / \mathrm{kg} /$ orally significantly increases the production of IL- 6 as well as improves the work of macrophages perform phagocytosis suggesting that honey can boost the immune system [20]. In contrast,

Table 3 Pre-intervention and post-intervention inflammatory markers in smokers

\begin{tabular}{llllll}
\hline & \multicolumn{2}{l}{ Smokers with honey $(n=32)$} & & \multicolumn{2}{l}{ Smokers without honey $(n=32)$} \\
\cline { 2 - 3 } & Pre-intervention & Post-intervention & & Pre-intervention & Post-intervention \\
\hline TNF-a $(\mathrm{pg} / \mathrm{mL})$ & $2615.86(145.31)$ & $3855.92(217.83)^{*}$ & $1.77(0.17)$ & $3026.64(128.74)$ & $2893.59(68.36)$ \\
IL-6 $(\mathrm{ng} / \mathrm{mL})$ & $1.60(0.19)$ & $1.61(0.26)^{*}$ & $1.96(0.26)$ & $1.73(0.26)$ \\
hsCRP $(\mathrm{ng} / \mathrm{mL})$ & $2.31(0.32)$ & $2.91(2.38)$ & $1.65(2.43)$ \\
\hline
\end{tabular}

Data are presented as mean (SEM). TNF-a: tumor necrosis factor-alpha; IL: interleukin; hsCRP: high sensitivity C-reactive protein ${ }^{*} p<0.05$ compared to the corresponding pre-intervention (Paired $t$-test) 
supplementation of honey at a dose of $70 \mathrm{~g}$ for 8 weeks to road cyclists significantly shows less elevation in seminal plasma IL-6 suggesting the beneficial effect of honey in reducing seminal plasma IL-6 [13]. In animal study, honey has also been shown to decrease the production of plasma IL-6 and is able to significantly suppress gene and protein expression of IL-6 in paw tissue of inflammatory-induced rat [21]. The inconsistency in these studies compared to the findings of the present study could be explained by the different type and dose of honey administration as well as could be due to the difference response between animal and human following honey supplementation.

In the present study, the level of plasma hsCRP at preintervention was significantly higher in smokers compared to non-smokers. This finding may suggest that cigarette smoke may induce vascular inflammatory response leading to atherothrombosis [22]. Following 12 weeks of honey supplementation, the result revealed a significant reduction in plasma level of hsCRP suggesting the anti-inflammatory effect of Tualang honey. Supplementation of antioxidant $\alpha$-tocopherol, which is present in honey, significantly reduces the levels of CRP in diabetic and non-diabetic patients [23, 24], thus may provide a protective effect against cardiovascular morbidity and mortality. In animal study, honey supplementation for 21 days at a dose of $1.0 \mathrm{~g} / \mathrm{kg}$ body weight significantly decreases CRP level in diabetic and hypercholesterol rats [25]. Furthermore, honey supplementation for 30 days significantly reduces the serum hsCRP among subjects with elevated hsCRP suggesting that supplementation of honey may reduce inflammation [26]. This is supported by previous study that phenolic compounds present in honey are responsible for the anti-inflammatory activity [27]. The suggested mechanism of action includes the suppression of proinflammatory activities of cyclooxygenase-2 and/or inducible nitric oxide synthase via these flavonoids [28]. Tualang honey has been reported to have flavonoids such as benzoic, gallic, syringic and trans-cinnamic acids and phenolic acids such as catechin and kaempferol [8] which may be responsible for the anti-inflammatory effect of honey as shown by the reduced hsCRP level among smokers and needs further study to evaluate its exact mechanism of action. However, the validity of this biomarker remains unclear as treatment with modified CRP and native CRP have been shown to give opposite effects on atherosclerosis in $\mathrm{ApoE}(-/-)$ mice [29].

\section{Conclusions}

In conclusion, chronic smokers had significantly higher levels of hsCRP compared to non-smokers at preintervention. Supplementation of honey for 12 weeks significantly increased the level of TNF- $\alpha$ but significantly reduced the level of hsCRP among chronic smokers. These conflicting findings on both these inflammatory markers may suggest that the effect of Tualang honey supplementation on inflammatory process among chronic smoker is inconclusive. Additional assessment on other inflammatory markers such as IL-1, IL-4 and IL-10 may be useful to give a better picture on the complex interplay between inflammatory and anti-inflamatory processes with larger number of subjects in future study.

\begin{abstract}
Abbreviations
CRP: C-reactive protein; hsCRP: High sensitivity C-reactive protein; IL6: Interleukin-6; IL-8: Interleukin-8; MAP: Mean arterial pressure; NF-kB: Nuclear factor kappa-light-chain-enhancer of activated B cells activation.;

SEM: Standard error of mean; TLR: Toll-like receptor; TNF-a: Tumor necrosis factor-a; USM: Universiti Sains Malaysia
\end{abstract}

\section{Funding}

This study has been supportd by Universiti Sains Malaysia Short Term Grant (Grant No:304/PPSP/61312025).

Availability of data and materials

The data are available for any scientific use with kind permission.

\section{Authors' contributions}

WSWG, ACR, and MM participated in the design of the study and drafted the manuscript. WSWG and MM participated in the statistical design and helped in the design of the study. WSWG and ACR recruited participants from clinics and communities. All authors had read and approved the final manuscript.

\section{Competing interest}

The authors declare that they have no competing interest.

\section{Consent for publication}

Not applicable.

\section{Ethics approval and consent to participate}

The study protocol and written informed consent were approved by Human Research Ethics Committee of Universiti Sains Malaysia (USM) (approval code: JEPeM [243.3.(6)]. The objectives of the study were carefully explained to the subjects and informed consent was taken from each subject. The study had strictly met the requirements of the Declaration of Helsinki.

\section{Publisher's Note}

Springer Nature remains neutral with regard to jurisdictional claims in published maps and institutional affiliations.

Received: 22 November 2016 Accepted: 23 March 2017

Published online: 28 March 2017

\section{References}

1. Ambrose JA, Barua RS. The pathophysiology of cigarette smoking and cardiovascular disease: an update. J Am CollCardiol. 2004;43:1731-7.

2. Petrescu F, Voican SC, Silosi S. Tumor necrosis factor-a serum levels in healthy smokers and nonsmokers. Int J Chron Obstruct Pulmon Dis. 2010;5:217-22.

3. Inoue T, Komoda H, Uchida T, Node K. Tropical fruit camu-camu (Myrciariadubia) has anti-oxidative and anti-inflammatory properties. J Cardiol. 2008;52:127-32.

4. Bogdanov S, Tomislav J, Sieber R, Gallmann P. Honey for nutrition and health: a review. J Am Coll Nutr. 2008;27:677-89.

5. Gheldof N, Wang XH, Engeseth NJ. Identification and quantification of antioxidant components of honeys from various floral sources. J Agric Food Chem. 2002;50:5870-7.

6. AI-Waili NS. Effects of daily consumption of honey solution on hematological indices and blood levels of minerals and enzymes in normal individuals. J Med Food. 2003;6:135-40.

7. Yao L, Jiang Y, D'Arcy B, Singanusong R, Datta N, Caffin N, et al. Quantitative high-performance liquid chromatography analyses of flavonoids in Australian eucalyptus honeys. J Agric Food Chem. 2004;52:210-4. 
8. Khalil MI, Alam N, Moniruzzaman M, Sulaiman SA, Gan SH. Phenolic acid composition and antioxidant properties of Malaysian honeys. J Food Sci. 2011;76:C921-8.

9. Michalkiewicz A, Biesaga M, Pyrzynska K. Solid-phase extraction procedure for determination of phenolic acids and some flavonols in honey. J Chromatogr A. 2008;1187:18-24.

10. Mohamed M, Sirajudeen KNS, Swamy M, Yaacob M, Sulaiman S. Studies on the antioxidant properties of Tualang honey of Malaysia. Afr J Tradit Complement Altern Med. 2010;7:59-63.

11. Ahmad I, Jimenez H, Yaacob NS, Yusuf N. Tualang honey protects keratinocytes from ultraviolet radiation induced inflammation and DNA damage. Photochem Photobiol. 2012;88:1198-204.

12. Prakash A, Medhi B, Avti PK, Saika UN, Pandhi P, Khanduja KL. Effect of different doses of Manuka honey in experimentally induced inflammatory bowel disease in rats. Phytother Res. 2008;22:1511-9.

13. Tartibian B, Maleki BH. The effects of honey supplementation on seminal plasma cytokines, oxidative stress biomarkers and antioxidants during 8 weeks of intensive cycling training. J Androl. 2012;33:449-61.

14. Gonçalves RB, Coletta RD, Silverio KG, Benevides L, Casati MZ, da Silva JS, et al. Impact of smoking on inflammation: overview of molecular mechanisms. Inflamm Res. 2011;60:409-24.

15. Tonks AJ, Dudley E, Porter NG, Parton J, Brazier J, Smith EL, et al. A 5.8-kDa component of manuka honey stimulates immune cells via TLR4. J Leukoc Biol. 2007:82:1147-55.

16. Kawai T, Akira S. Signaling to NF-KB by toll-like receptors. Trends Mol Med. 2007;13:460-9.

17. Helmersson J, Larsson A, Vessby B, Basu S. Active smoking and a history of smoking are associated with enhanced prostaglandin $\mathrm{F}$ (2-alpha), interleukin-6 and F2-isoprostane formation in elderly men. Atherosclerosis. 2005:81:201-7.

18. Tonks AJ, Cooper RA, Jones KP, Blair S, Parton J, Tonks A. Honey stimulates inflammatory cytokine production from monocytes. Cytokine. 2003;21:242-7.

19. Syam $Y$, Natsir R, Rahardjo SP, Usman AN, Dwiyanti R, Hatta M. Effect of Trigona honey to mRNA expression of interleukin-6 on Salmonella Typhi induced of BALB/c mice. Am J Microbiol Res. 2016:4:77-80.

20. Nikaein D, Khosravi AR, Moosavi Z, Shokri H, Erfanmanesh A, Ghorbani H, et al. Effect of honey as an immunomodulator against invasive aspergillosis in BALB/c mice. J Apic Res. 2014;53:84-90.

21. Hussein SZ, Mohd Yusoff K, Makpol S, Mohd Yusof YA. Gelam honey inhibits the production of proinflammatory, mediators NO, PGE2, TNF-a, and IL-6 in carrageenan-induced acute paw edema in rats. Evid Based Complement Alternat Med. 2012; ArticleID109636, http://dx.doi.org/10.1155/2012/109636.

22. Bermudez EA, Rifai N, Buring J, Manson JE, Ridker PM. Interrelationships among circulating interleukin-6, C-reactive protein, and traditional cardiovascular risk factors in women. Arterioscler Thromb Vasc Biol. 2002;22: 1668-73.

23. Patrick L, Uzick M. Cardiovascular disease: C-reactive protein and the inflammatory disease paradigm: HMG-CoA reductase inhibitors, alphatocopherol, red yeast rice, and olive oil polyphenols. A review of the literature. Altern Med Rev. 2001;6:248-71.

24. Jialal I, Devaraj S, Venugopal SK. Oxidative stress, inflammation, and diabetic vasculopathies: the role of alpha tocopherol therapy. Free Radic Res. 2002; 36:1331-6.

25. Asaduzzaman M, Sohanur Rahman M, Munira S, Muedur Rahman M, Hasan $\mathrm{M}$, et al. Effects of honey supplementation on hepatic and cardiovascular disease (CVD) marker in streptozotocin induced diabetic rats. J Diabetes Metab. 2015; 6:592.http//doi:10.4172/2155-6156.1000592

26. Yaghoobi N, Al-Waili N, Ghayour-Mobarhan M, Parizadeh SMR, Abasalti Z, Yaghoobi Z, et al. (2008). Natural honey and cardiovascular risk factors; effects on blood glucose, cholesterol, triacylglycerole, CRP, and body weight compared with sucrose. Sci World J. 2008:8:463-9.

27. Viuda-Martos M, Ruiz-Navajas Y, Fernandez-Lopez J, Perez-Alvarez JA. Functional properties of honey, propolis and royal jelly. J Food Sci. 2008;73:R117-24.

28. Cho H, Yun CW, Park WK, Kong JY, Kim KS, Park Y. Modulation of the activity of pro-inflammatory enzymes, COX-2 and iNOS, by chrysin derivatives. Pharmacol Res. 2004;49:37-43.

29. Schwedler SB, Amann K, Wernicke K, Krebs A, Nauck M, Wanner C, et al. Native C-reactive protein increases whereas modified $C$-reactive protein reduces atherosclerosis in apolipoprotein E-knockout mice. Circulation. 2005; 112:1016-23.

\section{Submit your next manuscript to BioMed Central and we will help you at every step:}

- We accept pre-submission inquiries

- Our selector tool helps you to find the most relevant journal

- We provide round the clock customer support

- Convenient online submission

- Thorough peer review

- Inclusion in PubMed and all major indexing services

- Maximum visibility for your research

Submit your manuscript at www.biomedcentral.com/submit
Biomed Central 